\title{
THERMAL ANALYSIS IN INDUSTRIAL ENVIRONMENTS
}

\author{
D. M. Price \\ Courtaulds Plc., 101 Lockhurst Lane, COVENTRY CV6 5RS, UK
}

\begin{abstract}
Common industrial procedures often expose polymers to liquids and vapors which may affect their thermal properties. By carrying out thermoanalytical measurements under simulated process conditions, the scientist can investigate environmental effects on the properties of the material. Such experiments can also be used to demonstrate the nature of processes taking place. This work illustrates case studies regarding the application of thermogravimetry, thermomechanical, dynamic mechanical and dielectric measurements to monitor the behaviour of fibers, films and adhesives under such conditions.
\end{abstract}

Keywords: adhesives, dyeing, fibers, photodegradation, spinning

\section{Introduction}

Good control of sample environment is a major requirement for thermal analysis. The investigator will often try to ensure that instruments are purged with a supply of dry, oxygen-free inert gas. In the real world, the interaction of materials with water vapor, the effect of residual solvents, washing and dyeing procedures, exposure to sunlight, etc. can all modify the behaviour of the sample. By simulating these conditions in the laboratory, the scientist can investigate how materials will perform during their processing and ultimate end-use. Four examples of the application of common thermoanalytical techniques to the characterization of polymeric systems will be given which illustrate the general utility of carrying out such measurements under "industrial environments".

\section{Examples}

\section{Production of Lyocell fibers}

The ability of amine oxides to directly solvate cellulose has been known for a number of decades, but the systematic development of a fiber spinning technology has only come about since the late 1970's [1]. Unlike the traditional viscose rayon process, the polymer remains underivatized and can be incorporated into solution at much higher concentrations. The resulting cellulose fibers are generically known as Lyocell and are recovered by precipitating the polymer from solution with water. Almost complete solvent recovery is possible resulting in an environmentally benign process. Courtaulds' fibers made by this route are marketed as Tencel ${ }^{\circledR}$ and are made by a "dry-jet/wet spinning" method whereby the polymer solution ("dope") is extruded through an array of fine holes ("spinning jet") at high temperature into an air gap and coagulated in an aqueous bath[2].

This stage is critical to the formation of the fiber and a study of the diffusion of water vapor into this material was undertaken using a modified thermobalance (TA Instruments model 951 TGA). The purge gas supply to the furnace was arranged so that dry air and

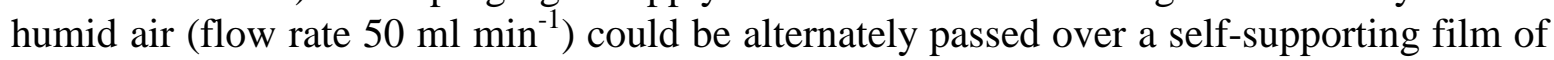
dope suspended from the arm of the balance. Moist air was generated by bubbling through a Dreshel bottle containing water, a second empty bottle being placed in-line served to prevent droplets of liquid being carried over into the oven. 
Figure 1 shows the mass versus time profile for a sample in the sequence moist, dry, moist and dry air. Two water sorbtion/desorption profiles are recorded. The data was fitted to the Crank's equation for diffusion into a semi-infinite plane sheet [3]:

$$
\frac{M_{t}}{M_{\infty}}=1-\sum_{n=0}^{n=\infty} \frac{8}{(2 n+1)^{2} \pi^{2}} \exp \left(\frac{-D(2 n+1)^{2} \pi^{2} t}{s^{2}}\right)
$$

where $M_{t}$ is the mass change at time $t, M_{\infty}$ the total mass change, $s$ the thickness to the film and $D$ the diffusion coefficient.

Figure 2 shows the trend in diffusion coefficient as a function of water content for consecutive sorption/desorbtion experiments. The diffusion coefficient was found to change only by a factor of two over the temperature range $30-90^{\circ} \mathrm{C}$ and water content range $12-35 \%$ weight. A single, averaged value could be used for modelling purposes and subsequent product improvement.

\section{Processing of cellulose acetate fibers}

Cellulose acetate fibers are produced by spinning a solution of the polymer in acetone into a heated air stream. The solvent evaporates to leave behind a bundle of filaments which are taken up and further processed into fibers and fabrics. The effects of residual solvent on the properties of the polymer are very important to its subsequent handling. Whilst there have been several studies conducted on the effects of low molecular weight molecules on cellulose and cellulose esters [4-7], these have been focused mainly on the effect of moisture on this class of hydrophilic polymer. Antiplasticization effects have also been observed whereby the stiffness of the polymer containing diluent is higher than the untreated material [5,7]. No data had been reported for the effects of acetone on cellulose acetate, and it was apparent that this area warranted investigation.

In order to study the effect of residual solvent on cellulose acetate, thermomechanical measurements on specially prepared solvent-cast films were made on a Mettler TMA 40 using the quartz film \& fiber extension accessory. Experiments were carried out under dynamic loading conditions of $\pm 0.05 \mathrm{~N}$ at $1 / 12 \mathrm{~Hz}$ superimposed on a static force of $0.1 \mathrm{~N}$ at a heating rate of $2^{\circ} \mathrm{C} \mathrm{min}^{-1}$. The furnace was purged directly with air (flow rate $25 \mathrm{ml}$ $\min ^{-1}$ ) introduced through a fine glass tube inserted into the top of the oven. For measurements using acetone saturated atmospheres, air was bubbled through the same gas line described in the previous example, substituting acetone for water. The raw instrumental output from the TMA was analysed to determine the average sample length and amplitude of the length change over a moving window of twelve data points collected over one complete loading/unloading cycle. The dynamic stress $(\sigma)$ on the sample is given by the change in load $(0.1 \mathrm{~N})$ divided by the cross sectional area of the specimen. The corresponding strain $(\varepsilon)$ is similarly given by the change in length (amplitude) divided the average length. The dynamic modulus ( $\left.\mathrm{E}^{*}\right)$ is obtained from ratio $\sigma / \varepsilon[8]$.

Plots of dynamic moduli for cellulose acetate films run under dry air and in the presence of acetone vapor are shown in Fig. 3. Room temperature moduli were within $\pm 4 \%$ of that obtained by conventional tensile testing. At room temperature, acetone lowers the dynamic modulus of the polymer, whilst between $115 \& 165^{\circ} \mathrm{C}$ the stiffness of the acetone-saturated film is higher than the untreated material. The softening point of the acetone containing film is lowered indicating a reduction in $\mathrm{T}_{\mathrm{g}}$ of the polymer by this material. Seymour et al.[5] have observed similar behavior for cellulose acetate with certain esters which they 
attribute to the additive giving rise to a new damping peak ( $\left.\beta^{\circ}\right)$ below the main $\alpha$ - or glass transition and the suppression of the $\gamma$-relaxation of the polymer. The increased modulus is limited to a narrow temperature range and at higher temperature the material will appear to be plasticized once more. This effect indicates that control of residual solvent may be critical to the processing of cellulose acetate.

\section{Carrier dyeing of acrylic fibers}

The dyeing and washing behavior of regenerated and synthetic fibers are markedly dependent upon temperature. For example, acrylic fibers must be dyed with cationic dyes above their $T_{g}$ in order to facilitate dye diffusion; in contrast, the characteristic high wet fastness properties of the resultant dyeings can be attributed primarily to the relative absence of dye diffusion that results from such aqueous treatments (eg. laundering) being carried out at temperatures below the $T_{g}$ of the fiber. Although the effect of temperature on the physical properties of dry fibers has been well documented, relatively little work has been carried out under dyebath (i.e. wet) conditions owing to practical difficulties that attend its measurement. Of practical interest was the effect of "carriers" - additives to the dyebath which are used to accelerate the rate of dye diffusion within the hydrophobic fibers. The mechanism by which these materials enhance dye uptake is thought to be by plasticizing the fiber, thus increasing the segmental mobility of the polymer chains [9].

Figure 4 shows the enhancement in dye uptake that can be achieved by incorporating various carriers into the dyebath. The increase in coloration reached a maximum at the solubility limit of the additive in the dyebath [10] and corresponded to the amount of carrier absorbed by the fiber. Using a dynamic mechanical technique whereby a bundle of fibers were heated under simulated dyebath conditions [11], the glass transition temperature of the wet polymer (normally $72^{\circ} \mathrm{C}$ in water alone) was measured in the presence of a variety of different additives. When these results were plotted against the increase in dye uptake (figure 5) it confirmed that the carriers were acting as a plasticizers and that a series of similar compounds all followed the same trend.

\section{Weathering of auto-tint window film adhesives}

Tinted self-adhesive plastic films are a popular means of limiting the effects of sunlight on the interiors of buildings and vehicles. Stuck to the inside surfaces of windows, these are used to filter infra-red and ultra-violet (UV) radiation to avoid heat build-up in offices and vehicles and fading of interior upholstery. After the glass itself, the adhesive is first to receive solar radiation and must be stabilized against photodegradation by appropriate choice of polymer and stabilizer package. This not only protects the adhesive but, by blocking short wavelength radiation, suppresses fading of the dyes used to color the film. The performance of candidate systems is assessed by exposure to intense radiation equivalent to the solar spectrum in a hot, humid environment during accelerated ageing in a Xenon weatherometer. Measurements of the UV transmission of the film are made at regular intervals in addition to critical evaluation of the optical appearance of the plate by eye. Since these products are to be used in vehicles, any blemishes in the film brought about by degradation of the adhesive are extremely undesirable.

Previous studies have demonstrated that dielectric thermal analysis is a powerful means of characterization of thin films of adhesive with a minimum of sample preparation [12]. This technique has also been used to study the UV degradation of poly(vinyl chloride) and 
poly(vinyl butryal) $[13,14]$. Dielectric measurements are highly sensitive to detecting changes in molecular mobility due to the irradiation process. Developments in instrumentation combined with the use of single-surface interdigitated electrodes [15] meant that it was possible to consider real-time monitoring of the properties of the adhesive during weathering.

In this study, test panels of window film were mounted on $3 \times 5 "$ glass plates for exposure in an Atlas Ci65 Xenon Weatherometer. A small window, the size of a singlesurface guarded IDEX sensor, was cut out of the PET film and sensor applied to the exposed surface of the adhesive after chilling the plate in a freezer to aid removal of the backing. Measurements of dielectric loss and permittivity were carried out from $0.1 \mathrm{~Hz}$ to $100 \mathrm{kHz}$ in decade steps at ambient temperature before and after $600 \& 1200 \mathrm{hr}$ exposure.

Table 1. Dielectric loss factor, e" $(0.1 \mathrm{~Hz})$ for adhesive with different stablizer pacakges during accelerated weathering

\begin{tabular}{llll}
\hline Sample & "standard" & "poor" & "good” \\
\hline initial & 3.00 & 2.86 & 3.14 \\
after $600 \mathrm{hr}$ & 8.59 & 12.6 & 3.18 \\
after $1200 \mathrm{hr}$ & 10.4 & 71.0 & 6.45
\end{tabular}

Values of dielectric loss, e", at $0.1 \mathrm{~Hz}$ for the same adhesive containing three candidate stabilizer packages are given in table 1 at different stages of weathering. The data indicates that, of the three stabilizer formulations, the "poor" package showed the largest increase in e". UV transmission measurements showed that, although starting with the same value $(\approx 0.4 \%)$, the transmission of "standard" package rises to $5.7 \%$ compared to around $2.5 \%$ for both adhesives containing the "good" and "poor" formulations after 1200 hours weathering. The "poor" sample exhibited severe optical distortion of the film, but remained light-fast, the "good" sample (the same formulation as the "poor" sample with an additional stabilizer) maintained good UV absorbtion and showed no blemishes. Even after 600 hours exposure, it was apparent that changes in adhesive behavior could be detected by this technique before deterioration in appearance could be seen by eye.

\section{Conclusions}

The four case studies above show the type of useful information that can be obtained by carrying out thermal analysis under the conditions that materials experience during their processing and end-use. The thermobalance is an ideal instrument for diffusion measurements where the uptake/loss of diffusant can be directly monitored by weight changes in the sample. Thermomechanical and dynamic mechanical measurements are useful for studying the change in stiffness and glass transition temperature brought about by the incorporation of potential plasticizers in the polymer matrix. Finally, measurement of the dielectric properties of thin films can be used to assess the resistance of adhesives to photodegradation. The author hopes that more workers will adopt these strategies for investigating the properties of materials under "industrial environments". 


\section{References}

1 US Patent 4,246,221 (1981).

2 H. A. Coulsey and S. B. Smith, Lenzinger Berichte, 75 (1996) 51.

3 J. Crank, The Mathematics of Diffusion, 2nd edition, Oxford University Press 1975, p. 48.

4 R.F. Eaton, T. H. Tran, M. Shen, T. F. Schatzki and E. Menefee, Polymer Preprints, 17 (1976) 54.

5 R. W. Seymour, S. Weinhold and S. K. Haynes, J. Macromol. Sci. Phys., B16 (1979) 337.

6 M. Scandola and G. Ceccrulli, Polymer, 26 (1985) 1953.

7 M. Scandola and G. Ceccrulli, Polymer, 26 (1985) 1958.

8 R. Resien, G. Widmann and R. Truttmann, Thermochim. Acta, 272 (1996) 27.

9 W. Ingamells, R. H. Peters \& S. R .Thornton, J.Appl. Polym. Sci., 17 (1973) 3733.

10 D. Aitken, S. M. Burkinshaw \& D. M. Price, Dyes \& Pigments, 18 (1992) 23.

11 D. Aitken, S. M. Birkinshaw, J. Catherall, R. Cox, R. E. Litchfield, D. M. Price and N. G. Todd, J. Appl. Polym. Sci., Appl. Polym. Symp., 47 (1991) 263.

12 P. J. Haines, Thermal Methods of Analysis, Blackie Academic \& Professional Press, London 1995, p. 228.

13 E. El-Shafee and G. R. Saad, Polym. Degr. Stab., 43 (1994) 315.

14 G. R. Saad, E El-Safee and M. W. Sabaa, Polym. Degr. Stab., 47 (1995) 209.

15 D. R. Day and D. D. Shepard, J. Coatings Technol., 60 (1988) 760, 57. 


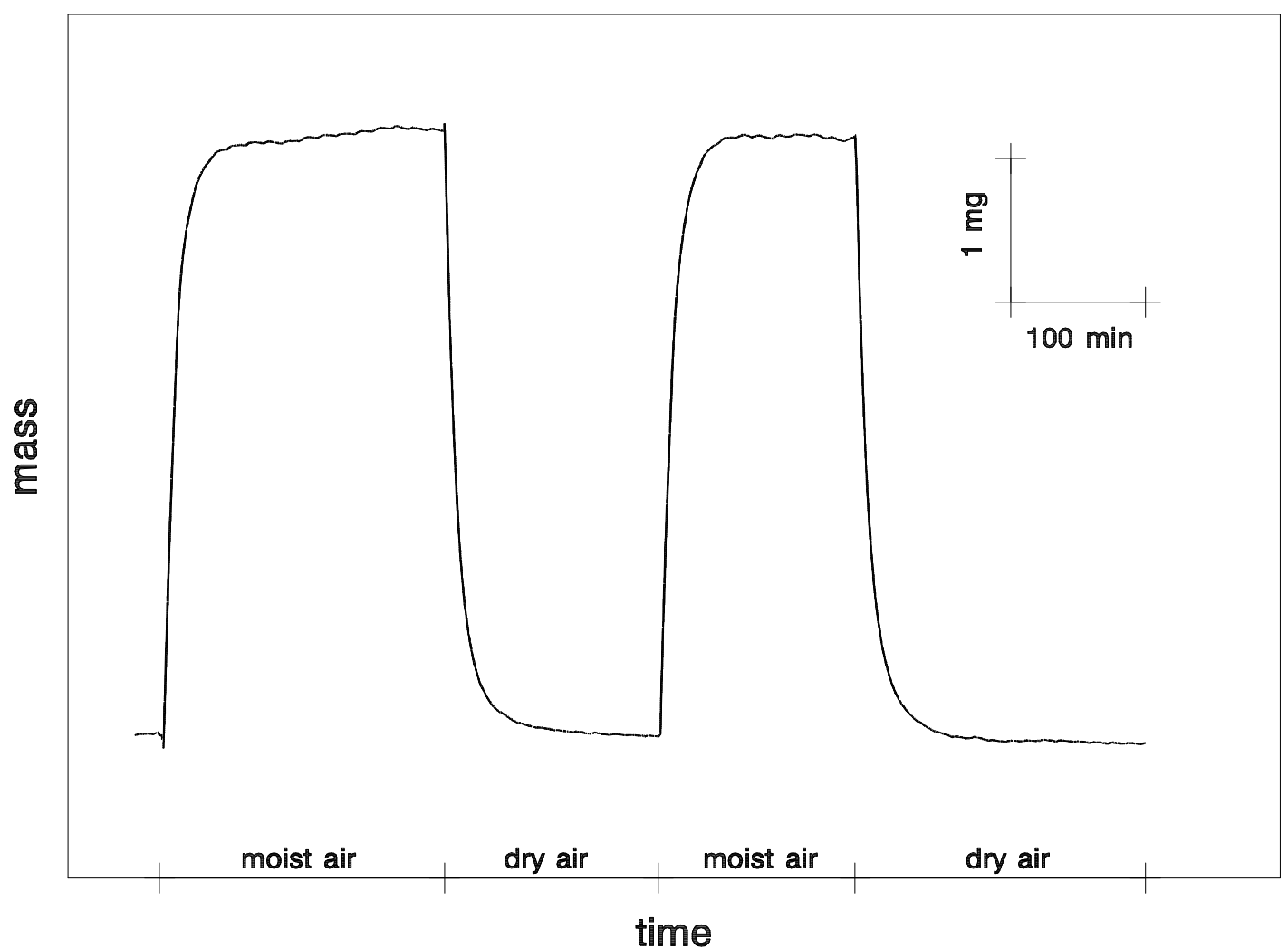

Fig. 1. Mass change of a thin film of Tencel ${ }^{\circledR}$ dope exposed to different humidity levels at $60^{\circ} \mathrm{C}$. 


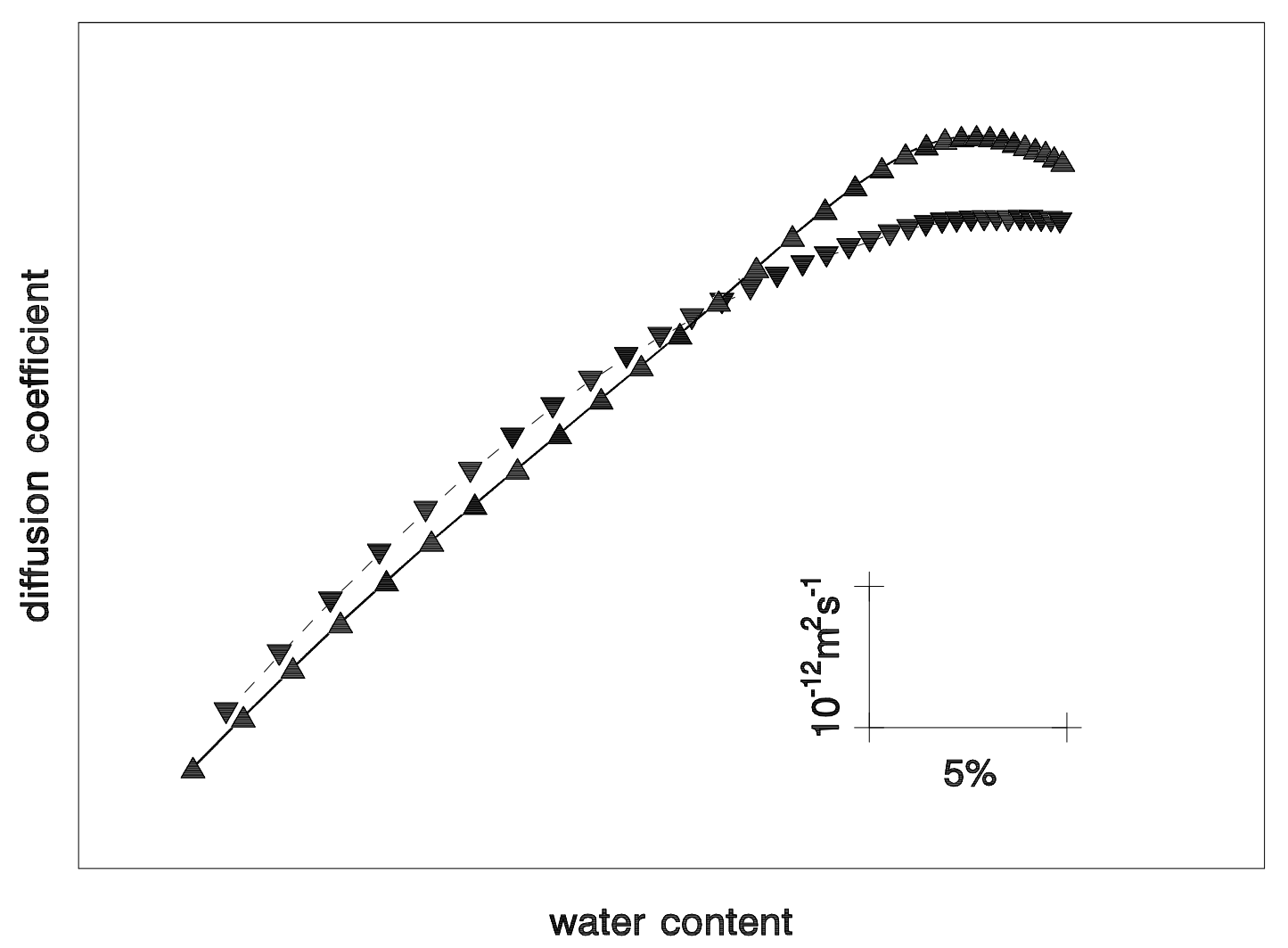

Fig. 2. Diffusion coefficient for water vapor into Tencel ${ }^{\circledR}$ dope as a function of water content calculated from the first sorbtion $(\boldsymbol{\Delta})$ and desorbtion $(\boldsymbol{\nabla})$ cycle in Fig. 1. 


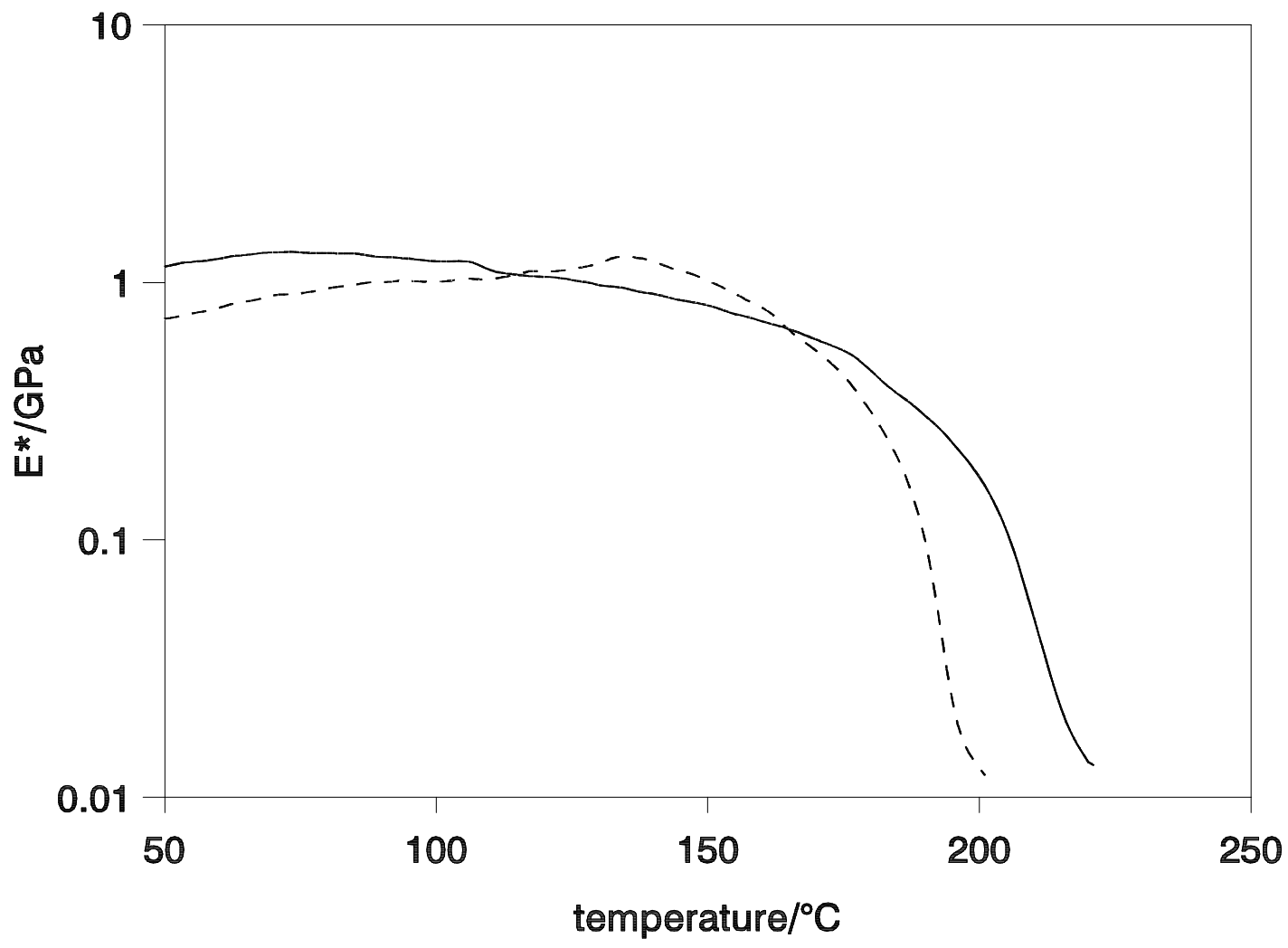

Fig. 3. Dynamic modulus, $E^{*}$, for cellulose acetate (solid line - dry, dashed line conditioned in acetone vapor) 


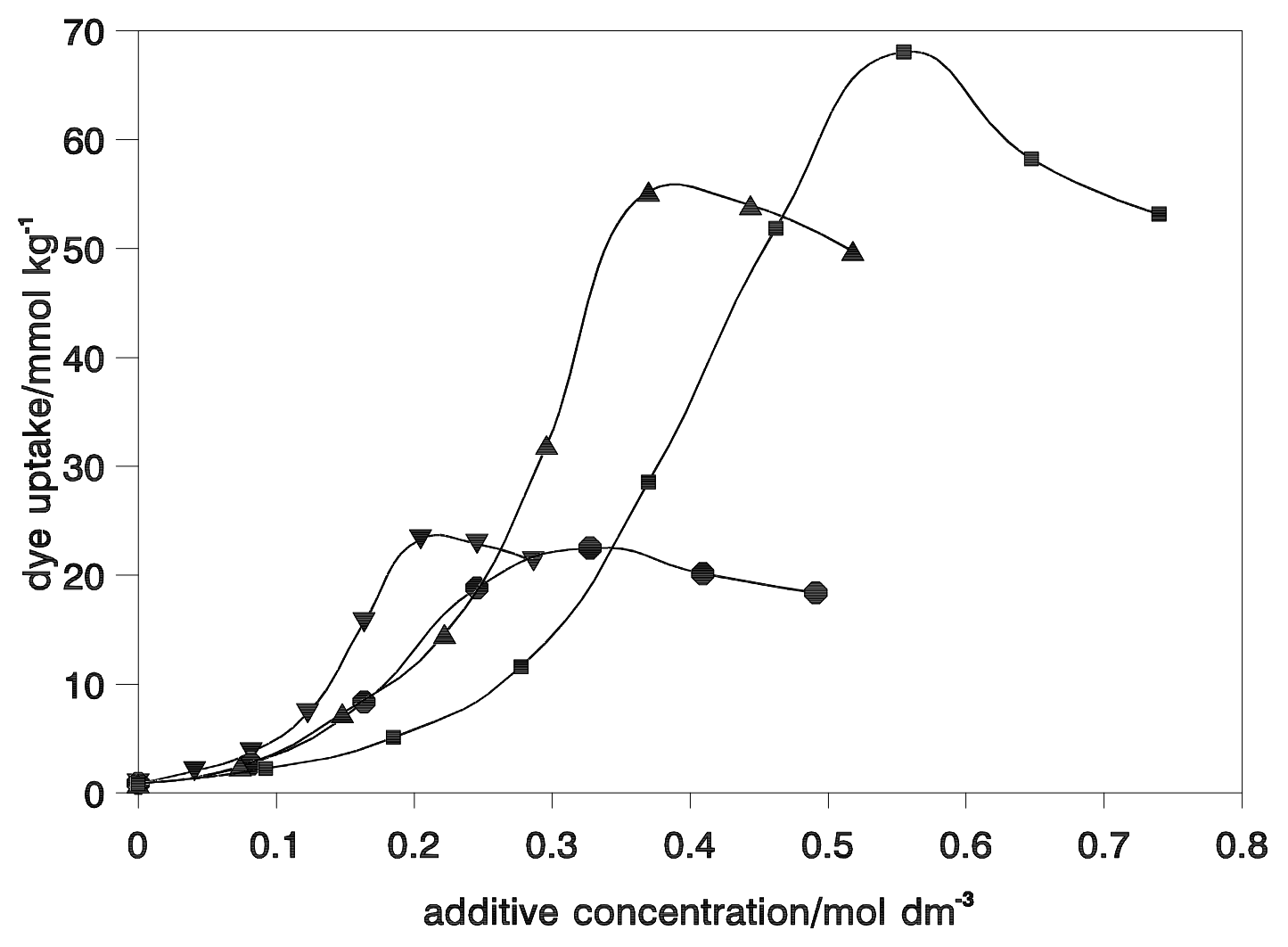

Fig. 4. Uptake of dye (CI Basic Blue 3) by Courtelle ${ }^{\circledR}$ acrylic fiber for 30 minute dyeings at $80^{\circ} \mathrm{C}$ with different dye bath additives ( $\boldsymbol{\square}$ - benzyl alcohol, $\boldsymbol{\Delta}$ - N-methyl formanilide, - phenylethyl alcohol, $\boldsymbol{\nabla}$-4-methylbenzyl alcohol). 


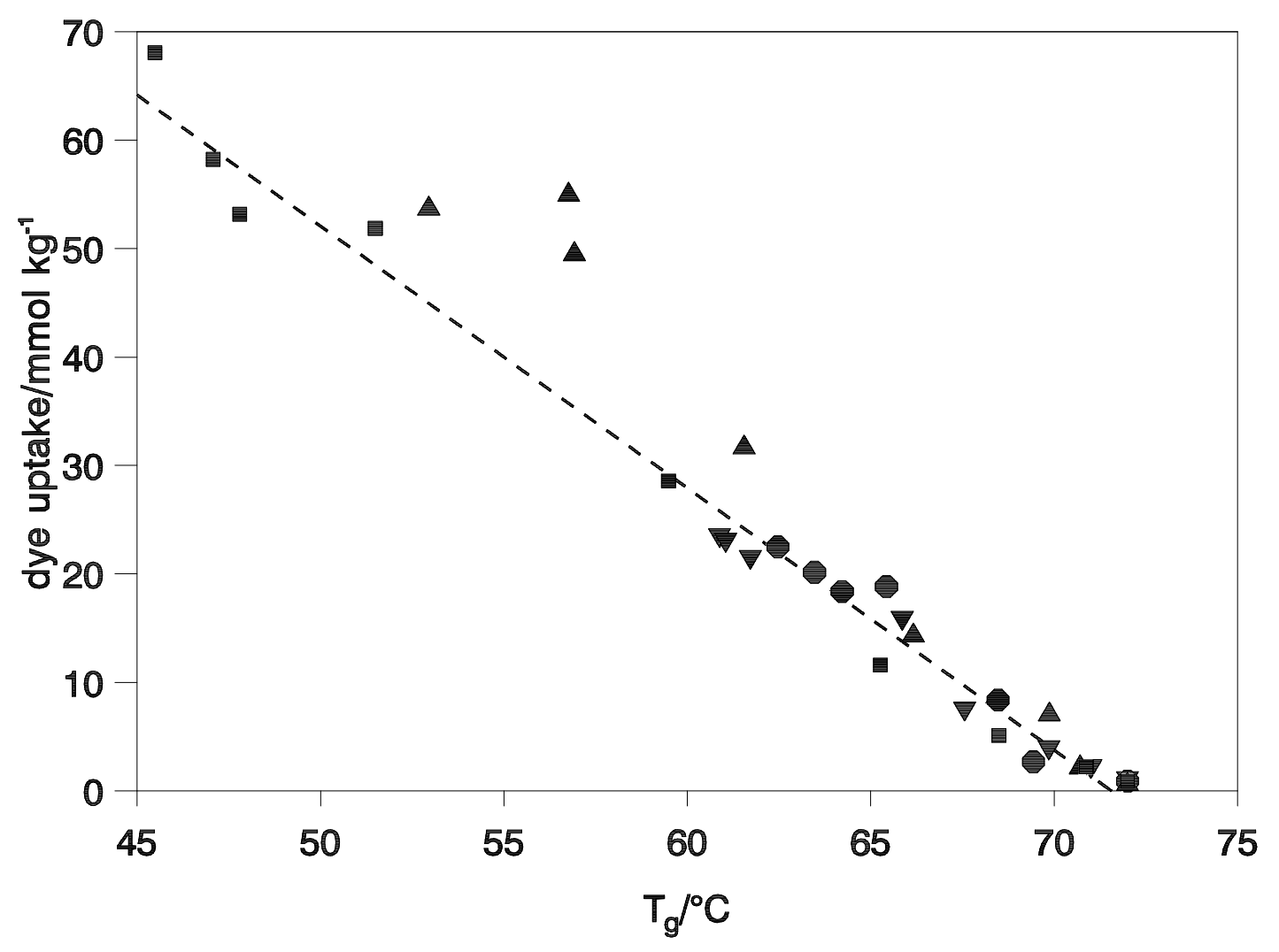

Fig. 5. Relationship between glass transition temperature ( $\mathrm{Tg}$ ) measured under dyebath and dye uptake with different dye bath additives ( $\mathbf{\square}$ - benzyl alcohol, $\boldsymbol{\Delta}$ - N-methyl formanilide, - phenylethyl alcohol, $\boldsymbol{\nabla}$ - 4-methylbenzyl alcohol). Dashed line indicates trend for the aromatic alcohols. 